\title{
GENERAL CONTRACTIVE MAPPING CONDITION OF FUZZY METRIC SPACES IN FIXED POINT THEOREM
}

\author{
${ }^{1}$ Ramasamy Mohan Raj and ${ }^{2}$ Veerachamy Malliga Devi \\ ${ }^{1}$ Department of Mathematics, Faculty of Science and Humanities, \\ Anna University Tirunelveli Region, Tirunelveli, Tamilnadu, India \\ ${ }^{2}$ Department of Mathematics, Faculty of Science and Humanities, \\ Anna University, Tuticorin Campus, Tamil Nadu, India
}

Received 2013-04-09; Revised 2014-02-04; Accepted 2014-02-06

\begin{abstract}
In several ways, related fixed point theorems on two or three metric spaces have been demonstrated. By applying contractive condition of integral type for class of weakly compatible maps in uncompleted intuitionistic fuzzy metric spaces without considering any continuous mappings, in this paper, we verify some frequent fixed point theorems for different mappings.
\end{abstract}

Keywords: Fuzzy Metric Space, Fixed Point Theorem, Banach Fixed Theorem, Mapping

\section{INTRODUCTION}

In 1922, Banach a polish mathematician proved a theorem under appropriate conditions and showed the existence and uniqueness of a fixed point this result is called Banach fixed point theorem. This theorem is also applied to prove the existence and uniqueness of the solutions of differential equations. Many authors have made different generalization of Banach fixed theorem. There are so many researches are available on this fixed point theorems. Normally, fixed point theory is classified into 3 categories such as (1) Topological Fixed point theory (2) Metric Fixed point theory and (3) Discrete fixed point theory. In these three areas, the boundary lines can be detected by employing the theorems such as (1) Brouwer's Fixed Point Theorem (2) Banach' s Fixed Point Theorem (3) Tarski' s Fixed Point Theorem.

The concept of Fuzzy set as a new way to represent vagueness in our everyday life (Zadeh, 1965). However, when the uncertainty is due to fuzziness rather than randomness, as sometimes in the measurement of an ordinary length, it seems that the concept of a fuzzy metric space is more suitable. We can divide them into following two groups: The first group involves those results in which a fuzzy metric on a set $X$ is treated as a map where $\mathrm{X}$ represents the totality of all fuzzy points of a set and satisfy some axioms which are analogous to the ordinary metric axioms. Thus, in such an approach numerical distances are set up between fuzzy objects. On the other hand in second group, we keep those results in which the distance between objects is fuzzy and the objects themselves may or may not be fuzzy (Manro et al., 2012).

\subsection{Preliminaries}

Some preliminary definitions are given below.

\section{Definition 1.1}

Zadeh (1965) A fuzzy set A in $X$ is a function with domain $\mathrm{X}$ and values in $[0,1]$.

\section{Definition 1.2}

Schweizer and Sklar (1960)A binary operation *: [0, $1] \times[0,1] \rightarrow[0,1]$ is a continuous t-norms if $*$ is satisfying conditions:

- $\quad *_{\text {is }}$ an commutative and associative

- $\quad *$ is continuous

- $\mathrm{a}^{*} 1=\mathrm{a}$ for all $\mathrm{a} \in[0,1]$

- $a^{*} b \leq c^{*} d$ whenever $a \leq c$ and $b \leq d$ and $a, b, c, d \in[0$, 1]

Corresponding Author: Veerachamy Malliga Devi, Department of Mathematics, Faculty of Science and Humanities, Anna University (Tuticorin Campus), Tamil Nadu, India 


\section{Definition 1.3}

George and Veeramani (1994) a 3-tuple (X, M, *) is said to be a fuzzy metric space if $\mathrm{X}$ is an arbitrary set, * is a continuous t-norm and $\mathrm{M}$ is a fuzzy set on $\mathrm{X} 2 \times[0$, $\infty$ ] satisfying the following conditions, for all $\mathrm{x}, \mathrm{y}, \mathrm{z} \in \mathrm{X}$, such that $\mathrm{t}$ is in $[0, \infty]$ :

- (f1) $M(x, y, t)>0$

- (f2) $M(x, y, t)=1$ if and only if $x=y$

- (f3) $M(x, y, t)=M(y, x, t)$

- (f4) $\mathrm{M}(\mathrm{x}, \mathrm{y}, \mathrm{t}) * \mathrm{M}(\mathrm{y}, \mathrm{z}, \mathrm{s}) \leq \mathrm{M}(\mathrm{x}, \mathrm{z}, \mathrm{t}+\mathrm{s})$

- (f5) $\mathrm{M}(\mathrm{x}, \mathrm{y}, *):(0, \infty) \rightarrow(0,1]$ is continuous

Then $M$ is called a fuzzy metric on $X$. Then $M(x, y, t)$ denotes the degree of nearness between $\mathrm{x}$ and $\mathrm{y}$ with respect to $t$.

\section{Definition 1.4}

Let $(\mathrm{X}, \mathrm{M}, *)$ is a fuzzy metric space:

- A sequence $\left\{x_{n}\right\}$ in $\mathrm{X}$ is said to be convergent to a point $x \in X$ (denoted by $\lim _{n \rightarrow \infty} x_{n}=x$ ) if, for all $t>0$, $\mathrm{n} \rightarrow \infty)$ :

$$
\lim _{n \rightarrow \infty} M\left(x_{n+p} 1 \times_{n} t\right)=1
$$

- A sequence $\left\{x_{n}\right\}$ in $X$ called a Cauchy sequence if, for all $t>0$ and $p>0$ :

$$
\lim _{n \rightarrow \infty} M\left(x_{n+p} 1 \times_{n} t\right)=1
$$

- A fuzzy metric space in which every Cauchy sequence is convergent is said to be complete

\section{Definition 1.5}

Rhoades (1988) a pair of self-mappings (f, g) of a fuzzy metric space $(\mathrm{X}, \mathrm{M}, *)$ is said to be:

- Weakly commuting if: $M(f g x, g f x, t) \geq M(f x, g x, t)$ $\forall \mathrm{x} \in \mathrm{X} \& \mathrm{t}>0$

- R-weakly commuting if there exists some $\mathrm{R}>0$ such that $M(f g x, g f x, t) \geq M(f x, g x, t / R) \forall x \in X$ and $t>0$

\section{Definition 1.6}

Jungck and Rhoades (2008) two self-mappings $f$ and $g$ of a fuzzy metric space $(\mathrm{X}, \mathrm{M}, *)$ are called compatible if:

$$
\lim _{n \rightarrow \infty} M\left(f g X_{n}, g f X_{n}, t\right)=1
$$

whenever $\left\{X_{n}\right\}$ is a sequence in $X$ such that:

$$
\lim _{n \rightarrow \infty} f X_{n}=\lim _{n \rightarrow \infty} g X_{n}=x \text { for some } x \text { in } X
$$

\section{Definition 1.7}

Grabiec (1988): Two self-maps $f$ and $g$ of a fuzzy metric space $(X, M, *)$ are called reciprocally continuous on $\mathrm{X}$ if:

$$
\operatorname{LimfgX}_{n \rightarrow \infty}=f x \text { and } \lim _{n \rightarrow \infty} g f X_{n}=g x
$$

whenever $\left\{X_{n}\right\}$ is a sequence in $X$ such that:

$$
\lim _{n \rightarrow \infty} \mathrm{fX}_{\mathrm{n}}=\lim _{\mathrm{n} \rightarrow \infty} \mathrm{gX}=\mathrm{x} \text { for some } \mathrm{x} \text { in } \mathrm{X}
$$

\section{Definition 1.8}

Let $X$ be a set, $f, g$ self maps of $X$. A point $x$ in $X$ is called a coincidence point of $\mathrm{f}$ and gifff $\mathrm{x}=\mathrm{gx}$. We shall call $w=f x=g x$ a point of coincidence of $f$ and $g$.

\section{Definition 1.9}

Jungck and Rhoades (2008) a pair of maps $\mathrm{S}$ and $\mathrm{T}$ is called weakly compatible pair if they commute at coincidence points.

\section{Definition 1.10}

Two self-maps $\mathrm{f}$ and $\mathrm{g}$ of a set $\mathrm{X}$ are occasionally weakly compatible (owc) iff there is a point $\mathrm{X}$ in $\mathrm{X}$ which is a coincidence point of $f$ and $g$ at which $f$ and $g$ commute.

\subsection{Literature Review}

Klim and Wardowski (2007) introduced the concept of contraction for set-valued maps in metric spaces and the conditions guaranteeing the existence of a fixed point for such a contraction are established. One of our results essentially generalizes the Nadler and Feng-Liu theorems and is different from the Mizoguchi-Takahashi result. The second result was different from the Reich and Mizoguchi-Takahashi results. The method which had used in the proofs of our results was inspired by Mizoguchi-Takahashi and Fen-Liu'sideas.

Mishra et al. (2010) proved some fixed point theorems for weakly compatible maps in fuzzy metric space satisfying integral type inequality but without assuming the completeness of the space or continuity of the mappings involved. Paper has extended this concept to fuzzy metric space and established the existence of common fixed points for a pair of self-mappings. The result obtained in the fuzzy metric space by using the 
notion of non-compatible maps or the property (E.A) were very interesting. Paper has proved common fixed point theorems for weakly compatible maps in fuzzy metric space by using the concept of (E.A) property, however, without assuming either the completeness of the space or continuity of the mappings involved.

Singh et al. (2012) aimed was to prove some common fixed point theorems in (GV)-fuzzy metric spaces. To prove the results, the research had employed the idea of compatibility. Wherein conditions on completeness of the underlying space (or subspaces) together with conditions on continuity in respect of any one of the involved maps were relaxed. Research results substantially generalized and improved a multitude of relevant common fixed point theorems of the existing literature in metric as well as fuzzy metric spaces which include some relevant results.

Bugajewski and Kasprzak (2009) proved a collection of new fixed point theorems forso-called weakly Fcontractive mappings. By analogy, paper has introduced a class of strongly F-expansive mappings and proved fixed point theorems for such mappings. Paper has also provided a few examples, which illustrated these results and, as an application, paper proved an existence and uniqueness theorem for the generalized Fredholm integral equation of the second kind. At last, paper has applied the Mönch fixed point theorem to prove two results on the existence of approximate fixed points of some continuous mappings.

Mishra and Choudhary (2010) presented some common fixed point theorems for occasionally weakly compatible mappings infuzzy metric spaces under various conditions.

Du (2012) discussed several characterizations of MTfunctions. Using the characterizations of MT-functions, paper established some existence theorems for coincidence point and fixed point in complete metric spaces. Results showed the new generalizations of Berinde-Berinde's fixed point theorem and MizoguchiTakahashi's fixed point theorem for nonlinear multivalued contractive maps.

Manro et al. (2012) in this study, we prove common fixed point theorems in fuzzy metric spaces. We also discuss result related to R-weakly commuting type mappings.

Chauhan (2009) proved a common fixed point theorem for two pairs of weakly compatible mappings in M-fuzzy metric spaces.

\subsection{Problem Definition Lemma 1}

Grabiec (1988): For all $\mathrm{x}, \mathrm{y} \in \mathrm{X}, \mathrm{M}(\mathrm{x}, \mathrm{y}, \bullet)$ is nondecreasing.

\section{Lemma 2}

Mishra et al. (1994) and Cho (1997): Let $\left\{\mathrm{y}_{\mathrm{n}}\right\}$ be a sequence in a fuzzy metric space $(\mathrm{X}, \mathrm{M}, *)$ with the condition (FM-6). If there exists a number $\mathrm{k} \in(0,1)$ such that $M\left(y_{n+2}, y_{n+1}, k t\right) M\left(y_{n+1}, y_{n}, t\right)$.

\section{Lemma 3}

Mishra et al. (1994): If for all $\mathrm{x}, \mathrm{y} \in \mathrm{X}, \mathrm{t}>0$ and for a number $\mathrm{k} \in(0,1) \mathrm{M}(\mathrm{x}, \mathrm{y}, \mathrm{kt})=\mathrm{M}(\mathrm{x}, \mathrm{y}, \mathrm{t})$.

\section{Theorem 4.1}

Let $(\mathrm{X}, \mathrm{M}, *)$ be a fuzzy metric space with continuous $\mathrm{t}$ norm $*$ defined by $\mathrm{t} * \mathrm{t} \geq \mathrm{t}$ for all $\mathrm{t} \in[0,1]$. Let $\mathrm{A}, \mathrm{B}, \mathrm{S}, \mathrm{T}, \mathrm{P}$ and $\mathrm{Q}$ be mappings from $\mathrm{X}$ into itself such that:

- $\mathrm{P}(\mathrm{X}) \subset \mathrm{AB}(\mathrm{X})$ and $\mathrm{Q}(\mathrm{X}) \subset \mathrm{ST}(\mathrm{X})$

- There exists a constant $\mathrm{k} \in(0,1)$ such that:

$$
\int_{0}^{\mathrm{M}(\mathrm{px}, \mathrm{Qy}, \mathrm{Kt})} \varphi(\mathrm{t}) \mathrm{dt} \geq \int_{0}^{\mathrm{m}(\mathrm{x}, \mathrm{y}, \mathrm{t})} \varphi(\mathrm{t}) \mathrm{dt}
$$

where, $\varphi: \mathrm{R}^{+} \rightarrow \mathrm{R}^{+}$is a Lebesque-integrable mapping which is summable, nonnegative and such that:

$$
\int_{0}^{\varepsilon} \varphi(t) d t>0 \text { for each } \varepsilon>0
$$

Where:

$$
m(x, y, t)=\min \left\{\begin{array}{l}
M(A B y, Q y, t) M(S T x, P x, t), \\
M(S T x, Q y, \alpha t), \\
M(A B y, P x,(2-\alpha) t), \\
M(A B y, S T x, t)
\end{array}\right\}
$$

For all $\mathrm{x}, \mathrm{y}, \in(0,2)$ and $\mathrm{t}>0$ and:

- If one of $\mathrm{P}(\mathrm{X}), \mathrm{AB}(\mathrm{X}), \mathrm{ST}(\mathrm{X})$ or $\mathrm{Q}(\mathrm{X})$ is a complete subspace of $\mathrm{X}$, then

- $\quad$ P and ST have a coincidence point

- $\mathrm{Q}$ and $\mathrm{AB}$ have a coincidence point Further, if

- $\mathrm{AB}=\mathrm{BA}, \mathrm{QB}=\mathrm{BQ}, \mathrm{QA}=\mathrm{AQ}, \mathrm{PT}=\mathrm{TP}, \mathrm{ST}=\mathrm{TS}$

- The pair $\{\mathrm{P}, \mathrm{ST}\}$ is weakly compatible, then

- $\mathrm{A}, \mathrm{B}, \mathrm{S}, \mathrm{T}, \mathrm{P}$ and $\mathrm{Q}$ have a unique common fixed point in $\mathrm{X}$

\section{Proof}

By (a), since $P(X) \subset A B(X)$, for any point $x_{0} \in X$, there exists a point $\mathrm{x}_{1} \in \mathrm{X}$ such that $\mathrm{Px}_{0}=\mathrm{ABx}_{1}$. Since $\mathrm{Q}(\mathrm{X}) \subset \mathrm{ST}(\mathrm{X})$, for this point $\mathrm{x}_{1}$ we can choose a point $\mathrm{x}_{2}$ 
$\in \mathrm{X}$ such that $\mathrm{Qx}_{1}=\mathrm{STx}_{2}$ and so on. Inductively, we can define a sequencein $\left\{y_{n}\right\}$ in $X$ such that for $n=0,1, \ldots$, :

$$
\begin{aligned}
& Y_{2 n}=\mathrm{Px}_{2 \mathrm{n}}=\mathrm{ABx}_{2 \mathrm{n}+1} \text { and } \\
& \mathrm{Y}_{2 \mathrm{n}+1}=\mathrm{Qx}_{2 \mathrm{n}+1}=\mathrm{STx}_{2 \mathrm{n}+2}
\end{aligned}
$$

By (b), for all $\mathrm{t}>0$ and $\alpha=1-\mathrm{q}$, with $\mathrm{q} \in(0,1)$, we have:

$$
\begin{aligned}
& \int_{0}^{\mathrm{M}(\mathrm{y} 2 \mathrm{n}+1, \mathrm{y} 2 \mathrm{n}+2, \mathrm{kt})} \varphi(\mathrm{t}) \mathrm{dt}=\int^{\mathrm{M}(\mathrm{Qx} 2 \mathrm{n}+1, \mathrm{P} \times 2 \mathrm{n}+2, \mathrm{kt})} \varphi(\mathrm{t}) \mathrm{dt} \\
& \quad=\int_{0}^{\mathrm{M}\left(\mathrm{Px}_{2 \mathrm{n}+2}, \mathrm{Px}_{2 \mathrm{n}+1}, \mathrm{kt}\right)} \varphi(\mathrm{t}) \mathrm{dt}, \geq \int_{0}^{\mathrm{M}\left(\mathrm{x}_{2 \mathrm{n}+2}, \times 2_{\mathrm{n}+1}, \mathrm{t}\right)} \varphi(\mathrm{t}) \mathrm{dt}
\end{aligned}
$$

$$
\mathrm{m}\left(\mathrm{x}_{2 \mathrm{n}+2}, \mathrm{x}_{2 \mathrm{n}+1}, \mathrm{t}\right)=\min
$$$$
\left\{\begin{array}{c}
\mathrm{M}\left(\mathrm{ABx}_{2 \mathrm{n}+1}, \mathrm{Qx}_{2 \mathrm{n}+1}, \mathrm{t}\right), \mathrm{M}\left(\mathrm{STx}_{2 \mathrm{n}+2}, \mathrm{Px}_{2 \mathrm{n}+2}, \mathrm{t}\right), \\
\mathrm{M}\left(\mathrm{STx}_{2 \mathrm{n}+2}, \mathrm{Qx}_{2 \mathrm{n}+1}, \alpha \mathrm{t}\right), \mathrm{M}\left(\mathrm{ABx}_{2 \mathrm{n}+1} \mathrm{Px}_{2 \mathrm{n}+2},(2-\alpha) \mathrm{t}\right), \\
\mathrm{M}\left(\mathrm{ABx}_{2 \mathrm{n}+1}, \mathrm{STx}_{2 \mathrm{n}+2}, \mathrm{t}\right)
\end{array}\right\}
$$

$$
=\min \left\{\begin{array}{l}
M\left(y_{2 n}, y_{2 n+1}, t\right), M\left(y_{2 n+1}, y_{2 n+2}, t\right), \\
M\left(y_{2 n+1}, y_{2 n+1}, \alpha t\right), M\left(y_{2 n}, y_{2 n+2},(1+q) t\right), \\
M\left(y_{2 n}, y_{2 n+1}, t\right)
\end{array}\right\}
$$

$$
\begin{aligned}
& \geq \min \left\{\begin{array}{l}
M\left(y_{2 n}, y_{2 n+1}, t\right), M\left(y_{2 n+1}, y_{2 n+2}, t\right), 1 \\
M\left(y_{2 n}, y_{2 n+1}, t\right), M\left(y_{2 n+1}, y_{2 n+2}, q t\right), \\
M\left(y_{2 n}, y_{2 n+1}, t\right)
\end{array}\right\} \\
& \geq \min \left\{\begin{array}{l}
M\left(y_{2 n}, y_{2 n+1}, t\right), M\left(y_{2 n+1}, y_{2 n+2}, t\right), \\
M\left(y_{2 n+1}, y_{2 n+2}, q t\right)
\end{array}\right\}
\end{aligned}
$$

Since the $\mathrm{t}$-norm $*$ is continuous and $\mathrm{M}(\mathrm{x}, \mathrm{y},$.$) is left$ continuous, letting $\mathrm{q} \rightarrow 1$ in (1a),we have:

$$
m\left(x_{2 n+2}, x_{2 n+1}, t\right) \geq \min \left\{\begin{array}{l}
M\left(y_{2 n}, y_{2 n+1}, t\right), \\
M\left(y_{2 n+1}, y_{2 n+2}, t\right) .
\end{array}\right\}
$$

Therefore:

$$
\int_{0}^{\mathrm{M}\left(\mathrm{y}_{2 \mathrm{n}+1}, \mathrm{y}_{2 \mathrm{n}+2}, \mathrm{kt}\right)} \varphi(\mathrm{t}) \mathrm{dt} \geq \int_{0}^{\min \mathrm{M}\left(\mathrm{y}_{2 \mathrm{n}+1}, \mathrm{y}_{2 \mathrm{n}+2}, \mathrm{t}\right), \mathrm{M}\left(\mathrm{y}_{2 \mathrm{n}+2}, \mathrm{y}_{2 \mathrm{n}+3}, \mathrm{t}\right)} \varphi(\mathrm{t}) \mathrm{dt}
$$

Similarly, we also have:

$$
\int_{0}^{\mathrm{M}\left(\mathrm{y}_{2 \mathrm{n}+2}, \mathrm{y}_{2 \mathrm{n}+3}, \mathrm{kt}\right)} \varphi(\mathrm{t}) \mathrm{dt} \geq \int_{0}^{\min \mathrm{M}\left(\mathrm{y}_{2 \mathrm{n}+1}, \mathrm{y}_{2 \mathrm{n}+2}, \mathrm{t}\right), \mathrm{M}\left(\mathrm{y}_{2 \mathrm{n}+2}, \mathrm{y}_{2 \mathrm{n}+3}, \mathrm{t}\right)} \varphi(\mathrm{t}) \mathrm{dt}
$$

In general, we have for $\mathrm{n}=1,2, \ldots$ :

$$
\int_{0}^{M\left(y_{n+1}, y_{n+2}, k t\right)} \varphi(t) d t \geq \int_{0}^{\min M\left(y_{n}, y_{n+1}, t\right), M\left(y_{n+1}, y_{n+2}, t\right)} \varphi(t) d t
$$

Consequently, it follows that for $n=1,2, \ldots, p=1$, 2...:

$$
\int_{0}^{M\left(y_{n+1}, y_{n+2}, k t\right)} \varphi(t) d t \geq \int_{0}^{\min M\left(y_{n}, y_{n+1}, t\right), M\left(y_{n+1}, y_{n+2}, t / k{ }^{p}\right)} \varphi(t) d t
$$

By noting that $\mathrm{M}\left(\mathrm{y}_{\mathrm{n}+1}, \mathrm{y}_{\mathrm{n}+2}, \mathrm{t} / \mathrm{k}^{\mathrm{p}}\right) \rightarrow 1$ as $\mathrm{p} \rightarrow \infty$, we have for $\mathrm{n}=1,2, \ldots \ldots$ :

$$
\int_{0}^{M\left(y_{n+1}, y_{n+2}, k t\right)} \varphi(t) d t \geq \int_{0}^{\min M\left(y_{n}, y_{n+1}, t\right)} \varphi(t) d t
$$

Hence by Lemma 2, $\left\{\mathrm{y}_{\mathrm{n}}\right\}$ is a Cauchy sequence. Now suppose $\mathrm{ST}(\mathrm{X})$ is complete. Note that the subsequence $\left\{\mathrm{y}_{2 \mathrm{n}+1}\right\}$ is contained in $\mathrm{ST}(\mathrm{X})$ and has a limit in $\mathrm{ST}(\mathrm{X})$. Call it $z$. Let $u \in S^{-1} z$. Then $S T u=z$. We shall use the fact that the subsequence $\left\{y_{2 n}\right\}$ also converges to $z$. By (b), we have:

$$
\begin{aligned}
& \int_{0}^{\mathrm{M}\left(\mathrm{Pu}, \mathrm{y}_{2 \mathrm{n}+1}, \mathrm{kt}\right)} \varphi(\mathrm{t}) \mathrm{dt}=\int_{0}^{\mathrm{M}\left(\mathrm{Pu}, \mathrm{Qx}_{2 \mathrm{n}+1}, \mathrm{kt}\right)} \varphi(\mathrm{t}) \mathrm{dt} \\
& \geq \int_{0}^{\mathrm{m}(\mathrm{u}, \mathrm{x} 2+1, \mathrm{t})} \varphi(\mathrm{t}) \mathrm{dt}
\end{aligned}
$$

Take $\alpha=1$ :

$$
\begin{gathered}
m\left(u, x_{2 n+1}, t\right)=\min \left\{\begin{array}{l}
M\left(A B x_{2 n+1}, Q x_{2 n+1}, t\right), M(S T u, P u, t), \\
M\left(S T u, Q x_{2 n+1}, t\right), M\left(A B x_{2 n+1}, P u, t\right), \\
M\left(A B x_{2 n+1}, S T u, t\right)
\end{array}\right\} \\
=\min \left\{\begin{array}{l}
M\left(y_{2 n}, y_{2 n+1}, t\right), M(z, P u, t), M\left(z, y_{2 n+1}, t\right) \\
M\left(y_{2 n}, P u, t\right), M\left(y_{2 n}, z, t\right)
\end{array}\right\}
\end{gathered}
$$

which implies that, as $\mathrm{n} \rightarrow \infty$ :

$$
\begin{array}{r}
\mathrm{m}\left(\mathrm{u}, \mathrm{x}_{2 \mathrm{n}+1}, \mathrm{t}\right)=\min \left\{\begin{array}{l}
1, \mathrm{M}(\mathrm{z}, \mathrm{Pu}, \mathrm{t}), 1, \mathrm{M} \\
(\mathrm{z}, \mathrm{Pu}, \mathrm{t}), 1
\end{array}\right\} \\
=\mathrm{M}(\mathrm{z}, \mathrm{Pu}, \mathrm{t})
\end{array}
$$

Therefore:

$$
\int_{0}^{\mathrm{M}(\mathrm{Pu}, \mathrm{z}, \mathrm{kt})} \varphi(\mathrm{t}) \mathrm{dt} \geq \int_{0}^{\mathrm{M}(\mathrm{Pu}, \mathrm{z}, \mathrm{t})} \varphi(\mathrm{t}) \mathrm{dt}
$$

Therefore, by Lemma 2.3, we have $\mathrm{Pu}=\mathrm{z}$. Since $\mathrm{STu}$ $=\mathrm{z}$ thus $\mathrm{Pu}=\mathrm{z}=\mathrm{STu}$, i.e., $\mathrm{u}$ is a coincidence point of $\mathrm{P}$ and ST. This proves (i). 
Since $\mathrm{P}(\mathrm{X}) \subset \mathrm{AB}(\mathrm{X}), \mathrm{Pu}=\mathrm{z}$ implies that $\mathrm{z} \in \mathrm{AB}(\mathrm{X})$. Let $v \in A B-1 z$. Then $A B v=z$. By (b), we have:

$$
\begin{aligned}
& \int_{0}^{\mathrm{M}\left(\mathrm{y}_{2 \mathrm{n}}, \mathrm{Qv}, \mathrm{kt}\right)} \varphi(\mathrm{t}) \mathrm{dt}=\int_{0}^{\mathrm{M}\left(\mathrm{Px}_{2 \mathrm{n}}, \mathrm{Qv}, \mathrm{kt}\right)} \varphi(\mathrm{t}) \mathrm{dt} \\
& \geq \int_{0}^{\mathrm{m}\left(\mathrm{x}_{2 \mathrm{n}}, \mathrm{v}, \mathrm{t}\right)} \varphi(\mathrm{t}) \mathrm{dt}
\end{aligned}
$$

Take $\alpha=1$ :

$$
\begin{aligned}
& m\left(x_{2 n}, v, t\right)= \\
& \min \left\{\begin{array}{l}
M(A B v, Q v, t), M\left(S T x_{2 n}, t\right) M\left(S T x_{2 n}, Q v, t\right), \\
M\left(A B v, P x_{2 n}, t\right), M\left(A B v, S T x_{2 n}, t\right)
\end{array}\right\} \\
& \quad=\min \left\{\begin{array}{l}
M(z, Q v, t), M\left(y_{2 n-1}, y_{2 n}, t\right), \\
M\left(y_{2 n-1}, Q v, t\right), \\
M\left(z, y_{2 n}, t\right), M\left(z, y_{2 n-1}, t\right)
\end{array}\right\}
\end{aligned}
$$

which implies that, as $\mathrm{n} \rightarrow \infty$ :

$$
\begin{aligned}
& \mathrm{m}\left(\mathrm{x}_{2 \mathrm{n}}+\mathrm{v}, \mathrm{t}\right)=\min \left\{\begin{array}{l}
\mathrm{M}(\mathrm{z}, \mathrm{Qv}, \mathrm{t}), 1, \\
\mathrm{M}(\mathrm{z}, \mathrm{Qu}, \mathrm{t}), 1,1
\end{array}\right\} \\
&=\mathrm{M}(\mathrm{z}, \mathrm{Qv}, \mathrm{t})
\end{aligned}
$$

Therefore:

$$
\int_{0}^{\mathrm{M}(\mathrm{z}, \mathrm{Qv}, \mathrm{kt})} \varphi(\mathrm{t}) \mathrm{dt} \geq \int_{0}^{\mathrm{M}(\mathrm{z}, \mathrm{Qv}, \mathrm{t})} \varphi(\mathrm{t}) \mathrm{dt}
$$

Therefore, by Lemma 2.3, we have $\mathrm{Qv}=\mathrm{z}$. Since $\mathrm{ABv}=\mathrm{z}$ thus $\mathrm{Qv}=\mathrm{z}=\mathrm{ABv}$, i.e., $\mathrm{v}$ is a coincidence point of $Q$ and $A B$. This proves (ii).

The remaining two cases pertain essentially to the previous cases. Indeed if $\mathrm{P}(\mathrm{X})$ or $\mathrm{Q}(\mathrm{X})$ is complete, then by (a) $\mathrm{z} \in \mathrm{P}(\mathrm{X}) \subset \mathrm{AB}(\mathrm{X})$ or $\mathrm{z} \in \mathrm{Q}(\mathrm{X}) \subset \mathrm{ST}(\mathrm{X})$. Thus (i) and (ii) are completely established.

Since the pair $\{\mathrm{P}, \mathrm{ST}\}$ is weakly compatible therefore $\mathrm{P}$ and ST commute at their coincidence point, i.e., $\mathrm{P}(\mathrm{STu})=(\mathrm{ST}) \mathrm{Pu}$ or Pz $=\mathrm{STz}$. By $(\mathrm{d})$, we have $\mathrm{Q}(\mathrm{ABv})$ $=(\mathrm{AB}) \mathrm{Qv}$ or $\mathrm{Qz}=\mathrm{ABz}$.

Now, we prove that $\mathrm{Pz}=\mathrm{z}$, by (b), we have:

$$
\begin{aligned}
& \int_{0}^{\mathrm{M}\left(\mathrm{Pz}, \mathrm{y}_{2 \mathrm{n}+1}, \mathrm{kt}\right)} \varphi(\mathrm{t}) \mathrm{dt} \geq \int_{0}^{\mathrm{M}(\mathrm{Pz}, \mathrm{Qx} 2 \mathrm{n}+1, \mathrm{kt})} \varphi(\mathrm{t}) \mathrm{dt} \\
& \geq \int_{0}^{\mathrm{m}\left(\mathrm{z}, \mathrm{x}_{2 \mathrm{n}+1}, \mathrm{t}\right)} \varphi(\mathrm{t}) \mathrm{dt}
\end{aligned}
$$

Take $\alpha=1$ :

$$
\begin{gathered}
m\left(z, x_{2 n+1}, t\right)=\min \left\{\begin{array}{l}
M\left(A B x_{2 n+1}, Q x_{2 n+1}, t\right), M(S T z, P z, t), \\
M\left(S T z, Q_{2 n+1}, t\right), M\left(A B x_{2 n+1}, P z, t\right) \\
M\left(A B x_{2 n+1}, S T z, t\right)
\end{array}\right\} \\
=\min \left\{\begin{array}{l}
m\left(y_{2 n}, y_{2 n+1}, t\right), M(P z, P z, t), \\
M p z, y_{2 n+1}, t, \\
M\left(y_{2 n}, P z, t\right), m\left(y_{2 n}, P z, t\right)
\end{array}\right\}
\end{gathered}
$$

Proceeding limit as $\mathrm{n} \rightarrow \infty$, we have:

$$
\begin{aligned}
& \mathrm{m}\left(\mathrm{z}, \mathrm{x}_{2 \mathrm{n}+2}, \mathrm{t}\right)=\min \left\{\begin{array}{l}
1,1, \mathrm{M}(\mathrm{Pz}, \mathrm{z}, \mathrm{t}), \mathrm{M} \\
\mathrm{z}, \mathrm{Pz}, \mathrm{t}), \mathrm{M}(\mathrm{z}, \mathrm{Pz}, \mathrm{t})
\end{array}\right\} \\
&=\mathrm{M}(\mathrm{Pz}, \mathrm{z}, \mathrm{t})
\end{aligned}
$$

Therefore:

$$
\int_{0}^{\mathrm{m}(\mathrm{P}, z, \mathrm{k}, \mathrm{t})} \varphi(\mathrm{t}) \mathrm{dt} \geq \int_{0}^{\mathrm{M}(\mathrm{P}, z, \mathrm{t}, \mathrm{t}} \varphi(\mathrm{t}) \mathrm{dt}
$$

Therefore, by Lemma 2.3, we have $\mathrm{Pz}=\mathrm{z}$ so $\mathrm{Pz}=$ $\mathrm{STz}=\mathrm{z}$. By (b), we have:

$$
\begin{aligned}
& \int_{0}^{\mathrm{m}\left(\mathrm{y}_{2 \mathrm{n}+2}, \mathrm{Qz}, \mathrm{kt}\right)} \varphi(\mathrm{t}) \mathrm{dt}=\int_{0}^{\mathrm{M}\left(\mathrm{Px}_{2 \mathrm{n}+2}, \mathrm{Qz}, \mathrm{kt}\right)} \varphi(\mathrm{t}) \mathrm{dt}, \\
& \geq \int_{0}^{\mathrm{m}\left(\mathrm{x}_{2 \mathrm{n}+2}, \mathrm{z}, \mathrm{t}\right)} \varphi(\mathrm{t}) \mathrm{dt}
\end{aligned}
$$

Take $\alpha=1$ :

$$
\begin{aligned}
m\left(x_{2 n+2}, z, t\right)= & \min \left\{\begin{array}{l}
M(A B z, Q z, t), M\left(S_{S_{2 n+2}}, P x_{2 n+2}, t\right), \\
M\left(S T x_{2 n+2}, Q z, t\right), m\left(A B z, P_{2 n+2}, t\right) \\
M\left(A B z, S T x_{2 n+2}, t\right)
\end{array}\right\} \\
= & \min \left\{\begin{array}{l}
M(Q z, Q z, t), M\left(y_{2 n+1}, y_{2 n+2}, t\right), \\
M\left(y_{2 n+1}, Q z, t\right), \\
M\left(Q z, y_{2 n+2}, t\right), M\left(Q z, y_{2 n+1}, t\right)
\end{array}\right\}
\end{aligned}
$$

Proceeding limit as $\mathrm{n} \rightarrow \infty$, we have:

$$
\begin{aligned}
\mathrm{m}\left(\mathrm{x}_{2 \mathrm{n}+2}, \mathrm{z}, \mathrm{t}\right) & =\min \left\{\begin{array}{l}
1,1, \mathrm{M}(\mathrm{z}, \mathrm{Qz}, \mathrm{t}), \\
\mathrm{M}(\mathrm{Qz}, \mathrm{z}, \mathrm{t}), \mathrm{M}(\mathrm{Qz}, \mathrm{z}, \mathrm{t})
\end{array}\right\} \\
= & \mathrm{M}(\mathrm{z}, \mathrm{Qz}, \mathrm{t}) .
\end{aligned}
$$

Therefore:

$$
\int_{0}^{\mathrm{M}(\mathrm{z}, \mathrm{Qz}, \mathrm{kt})} \varphi(\mathrm{t}) \mathrm{dt} \geq \int_{0}^{\mathrm{M}(\mathrm{z}, \mathrm{Qz}, \mathrm{t})} \varphi(\mathrm{t}) \mathrm{dt},
$$


Therefore, by Lemma 3, we have $\mathrm{Qz}=\mathrm{z}$ so $\mathrm{Qz}=$ $\mathrm{ABz}=\mathrm{z}$. By (b) and using (d), we have:

$$
\begin{aligned}
& \int_{0}^{\mathrm{M}(\mathrm{z}, \mathrm{Bz}, \mathrm{kt})} \varphi(\mathrm{t}) \mathrm{dt}=\int_{0}^{\mathrm{M}(\mathrm{Pz}, \mathrm{QBz}, \mathrm{kt})} \varphi(\mathrm{t}) \\
& \geq \int_{0}^{\mathrm{m}(\mathrm{z}, \mathrm{Bz}, \mathrm{t})} \varphi(\mathrm{t}) \mathrm{dt}
\end{aligned}
$$

Take $\alpha=1$ :

$$
\begin{aligned}
\mathrm{m}(\mathrm{z}, \mathrm{Bz}, \mathrm{t})=\min \left\{\begin{array}{l}
\mathrm{M}(\mathrm{AB}(\mathrm{Bz}), \mathrm{Q}(\mathrm{Bz}), \mathrm{t}), \mathrm{M}(\mathrm{STz}, \mathrm{Pz}, \mathrm{t}), \\
\mathrm{M}(\mathrm{STz}, \mathrm{Q}(\mathrm{Bz}), \mathrm{t}), \mathrm{M}(\mathrm{ABz}, \mathrm{Pz}, \mathrm{t}), \\
\mathrm{M}(\mathrm{AB}(\mathrm{Bz}), \mathrm{STz}, \mathrm{t})
\end{array}\right\} \\
=\min \{\mathrm{M}(\mathrm{Bz}, \mathrm{Bz}, \mathrm{t}), \mathrm{M}(\mathrm{z}, \mathrm{z}, \mathrm{t}), \mathrm{M}(\mathrm{z}, \mathrm{Bz}, \mathrm{t}),\} \\
=\min \{1,1, \mathrm{M}(\mathrm{z}, \mathrm{Bz}, \mathrm{t}), 1, \mathrm{M}(\mathrm{Bz}, \mathrm{z}, \mathrm{t})\} \\
=\mathrm{M}(\mathrm{z}, \mathrm{Bz}, \mathrm{t})
\end{aligned}
$$

Therefore:

$$
\int_{0}^{\mathrm{M}(\mathrm{z}, \mathrm{Bz}, \mathrm{kt})} \varphi(\mathrm{t}) \mathrm{dt} \geq \int_{0}^{\mathrm{M}(\mathrm{z}, \mathrm{Bz}, \mathrm{t})} \varphi(\mathrm{t}) \mathrm{dt} .
$$

Therefore, by Lemma 3, we have $\mathrm{Bz}=\mathrm{z}$. Since $\mathrm{ABz}=$ $\mathrm{z}$, therefore $\mathrm{Az}=\mathrm{z}$. Again by (b) and using (d), we have:

$$
\begin{aligned}
& \int_{0}^{\mathrm{M}(\mathrm{Tz}, \mathrm{z}, \mathrm{kt})} \varphi(\mathrm{t}) \mathrm{dt}=\int_{0}^{\mathrm{M}(\mathrm{P}(\mathrm{Tz}), \mathrm{Qz}, \mathrm{kt})} \varphi(\mathrm{t}) \mathrm{dt}, \\
& \geq \int_{0}^{\mathrm{m}(\mathrm{Tz}, \mathrm{z}, \mathrm{t})} \varphi(\mathrm{t}) \mathrm{dt},
\end{aligned}
$$

Take $\alpha=1$ :

$$
\begin{gathered}
\mathrm{m}(\mathrm{Tz}, \mathrm{z}, \mathrm{t})=\min \left\{\begin{array}{l}
\mathrm{M}(\mathrm{ABz}, \mathrm{Qz}, \mathrm{t}), \mathrm{M}(\mathrm{ST}(\mathrm{Tz}), \mathrm{P}(\mathrm{Tz}), \mathrm{t}), \\
\mathrm{M}(\mathrm{ST}(\mathrm{Tz}) \mathrm{Qz}, \mathrm{t}), \mathrm{M}(\mathrm{ABz}, \mathrm{p}(\mathrm{Tz}), \mathrm{t}), \\
\mathrm{M}(\mathrm{ABz}, \mathrm{ST}(\mathrm{Tz}), \mathrm{t})
\end{array}\right\} \\
=\min \left\{\begin{array}{l}
\mathrm{M}(\mathrm{Qz}, \mathrm{Qz}, \mathrm{t}), \mathrm{M}(\mathrm{Tz}, \mathrm{Tz}, \mathrm{t}), \\
\mathrm{M}(\mathrm{Tz}, \mathrm{z}, \mathrm{t}), \mathrm{M}(\mathrm{z}, \mathrm{Tz}, \mathrm{t}), \mathrm{M}(\mathrm{z}, \mathrm{Tz}, \mathrm{t})
\end{array}\right\} \\
=\min \{1,1, \mathrm{M}(\mathrm{Tz}, \mathrm{z}, \mathrm{t}), \mathrm{M}(\mathrm{z}, \mathrm{Tz}, \mathrm{t}), \mathrm{M}(\mathrm{z}, \mathrm{Tz}, \mathrm{t})\} \\
=\mathrm{M}(\mathrm{Tz}, \mathrm{z}, \mathrm{t}) .
\end{gathered}
$$

Therefore:

$$
\int_{0}^{\mathrm{M}(\mathrm{Tz}, \mathrm{z}, \mathrm{kt})} \varphi(\mathrm{t}) \mathrm{dt} \geq \int_{0}^{\mathrm{M}(\mathrm{Tz}, \mathrm{z}, \mathrm{t})} \varphi(\mathrm{t}) \mathrm{dt} .
$$

Therefore, by Lemma 3, we have $\mathrm{Tz}=\mathrm{z}$. Since $\mathrm{STz}=\mathrm{z}$, therefore $\mathrm{Sz}=\mathrm{z}$. By combining the above results, we have:

$$
\mathrm{Az}=\mathrm{Bz}=\mathrm{Sz}=\mathrm{Tz}=\mathrm{Pz}=\mathrm{Qz}=\mathrm{z}
$$

That is $\mathrm{z}$ is a common fixed point of $\mathrm{A}, \mathrm{B}, \mathrm{S}, \mathrm{T}, \mathrm{P}$ and $\mathrm{Q}$. The uniqueness of the common fixed point of A, B, S, T, P and $\mathrm{Q}$ follows easily from (b). This completes the proof.
If we put $\mathrm{P}=\mathrm{Q}$ in Theorem 4.1, we have the following result.

\section{Corollary 4.1:}

Let $(\mathrm{X}, \mathrm{M}, *)$ be a fuzzy metric space with continuous tnorm $*$ defined by $\mathrm{t} * \mathrm{t} \geq \mathrm{t}$ for all $\mathrm{t} \in[0,1]$. Let $\mathrm{A}, \mathrm{B}, \mathrm{S}, \mathrm{T}$ and $\mathrm{P}$ be mappings from $\mathrm{X}$ into itself such that:

- $\mathrm{P}(\mathrm{X}) \subset \mathrm{AB}(\mathrm{X})$ and $\mathrm{P}(\mathrm{X}) \subset \mathrm{ST}(\mathrm{X})$

- There exists a constant $\mathrm{k} \in(0,1)$ such that

$$
\int_{0}^{\mathrm{M}(\mathrm{Px}, \mathrm{Py}, \mathrm{kt})} \varphi(\mathrm{t}) \mathrm{dt} \geq \int_{0}^{\mathrm{m}(\mathrm{x}, \mathrm{y}, \mathrm{t})} \varphi(\mathrm{t}) \mathrm{dt} .
$$

where, $\varphi: \mathrm{R}^{+} \rightarrow \mathrm{R}^{+}$is a Lebesque-integrable mapping which is summable, nonnegative and such that:

$$
\int_{0}^{\varepsilon} \varphi(t) d t>0 \text { for each } \varepsilon>0
$$

Where:

$$
m(x, y, t)=\min \left\{\begin{array}{l}
M(A B y, P y, t), M(S T x, P x, t), M(S T x, \alpha t), \\
M(A B y, P x, 2-\alpha) t), M(A B y, S T x, t)
\end{array}\right\}
$$

For all $\mathrm{x}, \mathrm{y} \in \mathrm{X}, \alpha \in(0,2)$ and $\mathrm{t}>0$ and:

- If one of $\mathrm{P}(\mathrm{X}), \mathrm{AB}(\mathrm{X})$ or $\mathrm{ST}(\mathrm{X})$ is a complete subspace of $\mathrm{X}$, then

- $\quad \mathrm{P}$ and ST have a coincidence point

- $\quad \mathrm{P}$ and $\mathrm{AB}$ have a coincidence point Further, if

- $\mathrm{AB}=\mathrm{BA}, \mathrm{PB}=\mathrm{BP}, \mathrm{PA}=\mathrm{AP}, \mathrm{PT}=\mathrm{TP}, \mathrm{ST}=\mathrm{TS}$ and

- The pair $\{\mathrm{P}, \mathrm{ST}\}$ is weakly compatible, Then

- $\quad$ A, B, S, T and P have a unique common fixed point in $X$. If we put $B=T=I x$ (the identity mapping on $\mathrm{X}$ ) in Theorem 4.1, we have the following result:

\section{Corollary 4.2}

Let $(\mathrm{X}, \mathrm{M}, *)$ be a fuzzy metric space with continuous $\mathrm{t}$-norm $*$ defined by $\mathrm{t} * \mathrm{t} \geq \mathrm{t}$ for all $\mathrm{t} \in[0,1]$. Let $\mathrm{A}, \mathrm{S}, \mathrm{P}$ and $\mathrm{Q}$ be mappings from $\mathrm{X}$ into itself such that:

- $\mathrm{P}(\mathrm{X}) \subset \mathrm{A}(\mathrm{X})$ and $\mathrm{Q}(\mathrm{X}) \subset \mathrm{S}(\mathrm{X})$

- There exists a constant $\mathrm{k} \in(0,1)$ such that:

$$
\int_{0}^{\mathrm{M}(\mathrm{Px}, \mathrm{Qy}, \mathrm{kt})} \varphi(\mathrm{t}) \mathrm{dt} \geq \int_{0}^{\mathrm{m}(\mathrm{x}, \mathrm{y}, \mathrm{t})} \varphi(\mathrm{t}) \mathrm{dt}
$$

where, $\varphi: \mathrm{R}^{+} \rightarrow \mathrm{R}^{+}$is a Lebesque-integrable mapping which is summable, nonnegative and such that:

$$
\int_{0}^{\varepsilon} \varphi(t) d t>0 \text { for each } \varepsilon>0
$$


Where:

$$
m(x, y, t)=\min \left\{\begin{array}{l}
M(A y, Q y, t), M(S x, P x, t), M(S x, Q y, \alpha t), \\
M(A y, P x, 2-\alpha) t), M(A y, S x, t)
\end{array}\right\}
$$

For all $\mathrm{x}, \mathrm{y} \in \mathrm{X}, \alpha \in(0,2)$ and $\mathrm{t}>0$ and:

- If one of $\mathrm{P}(\mathrm{X}), \mathrm{A}(\mathrm{X}), \mathrm{S}(\mathrm{X})$ or $\mathrm{Q}(\mathrm{X})$ is a complete subspace of $X$

- then

- $\quad \mathrm{P}$ and $\mathrm{S}$ have a coincidence point and

- $\quad \mathrm{Q}$ and A have a coincidence point.

Further, if

- $\quad \mathrm{QA}=\mathrm{AQ}$ and

- The pair $\{\mathrm{P}, \mathrm{S}\}$ is weakly compatible, then

- A, S, P and Q have a unique common fixed point in $\mathrm{X}$

If we put $\mathrm{A}=\mathrm{S}$ in Corollary 4.2, we have the following result:

\section{Corollary 4.3}

Let $(\mathrm{X}, \mathrm{M}, *)$ be a fuzzy metric space with continuous defined by $\mathrm{t} * \mathrm{t} \geq \mathrm{t}$ for all $\mathrm{t} \in[0,1]$. Let $\mathrm{A}, \mathrm{P}$ and $\mathrm{Q}$ be mappings from $\mathrm{X}$ such that:

- $\mathrm{P}(\mathrm{X}) \subset \mathrm{A}(\mathrm{X})$ and $\mathrm{Q}(\mathrm{X}) \subset \mathrm{A}(\mathrm{X})$

- There exists a constant $\mathrm{k} \in(0,1)$ such that:

$$
\int_{0}^{\mathrm{M}(\mathrm{Px}, \mathrm{Qy}, \mathrm{kt})} \varphi(\mathrm{t}) \mathrm{dt} \geq \int_{0}^{\mathrm{m}(\mathrm{x}, \mathrm{y}, \mathrm{t})} \varphi(\mathrm{t}) \mathrm{dt}
$$

where, $\varphi: \mathrm{R}^{+} \rightarrow \mathrm{R}^{+}$is a Lebesque-integrable mapping which is summa nonnegative and such that:

$$
\int_{0}^{\varepsilon} \varphi(t) d t>0 \text { foreach } \varepsilon>0
$$

Where:

$$
m(x, y, t)=\min \left\{\begin{array}{l}
M(A y, Q y, t), M(S x, P x, t), M(S x, Q y, \alpha t), \\
M(A y, P x, 2-\alpha) t), M(A y, S x, t)
\end{array}\right\}
$$

For all $\mathrm{x}, \mathrm{y} \in \mathrm{X}, \alpha \in(0,2)$ and $\mathrm{t}>0$ and:

- If one of $\mathrm{P}(\mathrm{X}), \mathrm{Q}(\mathrm{X})$ or $\mathrm{A}(\mathrm{X})$ is a complete subspace of $X$, then

- $\quad$ P and A have a coincidence point and

- $\quad$ Q and A have a coincidence point Further, if

- $\quad \mathrm{QA}=\mathrm{AQ}$ and

- The pair $\{\mathrm{P}, \mathrm{A}\}$ is weakly compatible, then

- $\mathrm{A}, \mathrm{P}$ and $\mathrm{Q}$ have a unique common fixed point in $\mathrm{X}$
In Theorem 4.1, if we replace the condition $\mathrm{QA}=\mathrm{AQ}$ by weak compatibility of the pair $\{\mathrm{Q}, \mathrm{AB}\}$ then we have the following theorem.

\section{Theorem 4.2}

Let $(\mathrm{X}, \mathrm{M}, *)$ be a fuzzy metric space with continuous tnorm $*$ defined by $\mathrm{t} * \mathrm{t} \geq \mathrm{t}$ for all $\mathrm{t} \in[0,1]$. Let $\mathrm{A}, \mathrm{B}, \mathrm{S}, \mathrm{T}, \mathrm{P}$ and $\mathrm{Q}$ be mappings from $\mathrm{X}$ into itself such that:

- $\mathrm{P}(\mathrm{X}) \subset \mathrm{AB}(\mathrm{X})$ and $\mathrm{Q}(\mathrm{X}) \subset \mathrm{ST}(\mathrm{X})$

- There exists a constant $\mathrm{k} \in(0,1)$ such that:

$$
\int_{0}^{\mathrm{M}(\mathrm{Px}, \mathrm{Qy}, \mathrm{kt})} \varphi(\mathrm{t}) \mathrm{dt} \geq \int_{0}^{\mathrm{m}(\mathrm{x}, \mathrm{y}, \mathrm{t})} \varphi(\mathrm{t}) \mathrm{dt}
$$

where, $\varphi: \mathrm{R}^{+} \rightarrow \mathrm{R}^{+}$is a Lebesque-integrable mapping which is summable, nonnegative and such that:

$$
\int_{0}^{\varepsilon} \varphi(t) d t>0 \text { for each } \varepsilon>0
$$

Where:

$$
\begin{aligned}
& \mathrm{m}(\mathrm{x}, \mathrm{y}, \mathrm{t})= \\
& \min \left\{\begin{array}{l}
M(A B y, Q y, t), M(S T x, P x, t), M(S T x, Q y, \alpha t), \\
M(A B y, P x, 2-\alpha) t), M(A B y, S T x, t)
\end{array}\right\}
\end{aligned}
$$

For all $\mathrm{x}, \mathrm{y} \in \mathrm{X}, \alpha \in(0,2)$ and $\mathrm{t}>0$ and:

- If one of $\mathrm{P}(\mathrm{X}), \mathrm{AB}(\mathrm{X}), \mathrm{ST}(\mathrm{X})$ or $\mathrm{Q}(\mathrm{X})$ is a complete subspace of $\mathrm{X}$, then

- $\quad$ P and ST have a coincidence point

- $\quad$ Q and AB have a coincidence point Further, if

- $\mathrm{AB}=\mathrm{BA}, \mathrm{QB}=\mathrm{BQ}, \mathrm{PT}=\mathrm{TP}, \mathrm{ST}=\mathrm{TS}$

- The pairs $\{P, S T\}$ and $\{\mathrm{Q}, \mathrm{AB}\}$ are weakly compatible, Then

- $\quad \mathrm{A}, \mathrm{B}, \mathrm{S}, \mathrm{T}, \mathrm{P}$ and $\mathrm{Q}$ have a unique common fixed point in X. By using Theorem 4.2, we have the following theorem

\section{Theorem 4.3}

Let $(X, M, *)$ be a fuzzy metric space with continuous $\mathrm{t}$-norm $*$ defined by $\mathrm{t} * \mathrm{t} \geq \mathrm{t}$ for all $\mathrm{t} \in[0,1]$. Let $\mathrm{A}, \mathrm{B}, \mathrm{S}, \mathrm{T}$ and $\mathrm{Pi}$, for $\mathrm{i}=0,1,2, \ldots$, be mappings from $\mathrm{X}$ into itself such that:

- $\mathrm{P}_{0}(\mathrm{X}) \subset \mathrm{AB}(\mathrm{X})$ and $\mathrm{Pi}(\mathrm{X}) \subset \mathrm{ST}(\mathrm{X})$, for $\mathrm{i} \in \mathbb{N}$

- There exists a constant $k \in(0,1)$ such that:

$$
\int_{0}^{\mathrm{M}\left(\mathrm{P}_{0} \mathrm{x}, \mathrm{P}_{\mathrm{i}} \mathrm{y}, \mathrm{kt}\right)} \varphi(\mathrm{t}) \mathrm{dt} \geq \int_{0}^{\mathrm{m}(\mathrm{x}, \mathrm{y}, \mathrm{t})} \varphi(\mathrm{t}) \mathrm{dt}
$$


where, $\varphi: \mathrm{R}^{+} \rightarrow \mathrm{R}^{+}$is a Lebesque-integrable mapping which is summable, nonnegative and such that:

$$
\int_{0}^{\varepsilon} \varphi(t) d t>0 \text { for each } \varepsilon>0
$$

Where:

$$
\begin{aligned}
& m(x, y, t)= \\
& \min \left\{\begin{array}{l}
M\left(A B y, P_{i} y, t\right), M\left(S T x, P_{0} x, t\right), M\left(S T x, P_{i} y, \alpha t\right), \\
\left.M\left(A B y, P_{0} x, 2-\alpha\right) t\right), M(A B y, S T x, t)
\end{array}\right\}
\end{aligned}
$$

For all $\mathrm{x}, \mathrm{y} \in \mathrm{X}, \alpha \in(0,2)$ and $\mathrm{t}>0$ and:

- If one of $\mathrm{P}_{0}(\mathrm{X}), \mathrm{AB}(\mathrm{X})$ or $\mathrm{ST}(\mathrm{X})$ is a complete subspace of $X$ or alternatively, $P_{i}$, for $i \in \mathbb{N}$, are complete subspace of $\mathrm{X}$, then

- $\quad \mathrm{P}_{0}$ and ST have a coincidence point

- For $i \in \mathbb{N}, \mathrm{P}_{\mathrm{i}}$ and $\mathrm{AB}$ have a coincidence point Further, if

- $\mathrm{AB}=\mathrm{BA}, \mathrm{P}_{\mathrm{i}} \mathrm{B}=\mathrm{BP}_{\mathrm{i}}(\mathrm{i} \in \mathbb{N}), \mathrm{P} 0 \mathrm{~T}=\mathrm{TP} 0, \mathrm{ST}=\mathrm{TS}$

- The pairs $\left\{\mathrm{P}_{0}, \mathrm{ST}\right\}$ and $\left\{\mathrm{P}_{\mathrm{i}}(\mathrm{i} \in \mathbb{N}), \mathrm{AB}\right\}$ are weakly compatible, then

- $\mathrm{A}, \mathrm{B}, \mathrm{S}, \mathrm{T}$ and $\mathrm{P}_{\mathrm{i}}$, for $\mathrm{i}=0,1,2 \ldots$, have a unique common fixed point in $\mathrm{X}$

\section{CONCLUSION}

Some conditions involve linear and nonlinear expressions (rational, irrational and of general type). Recently, some fixed point results for mappings satisfying an integral type contractive condition. Fixed point theorems for various generalizations of contraction mappings in probabilistic and fuzzy metric space were described. In our proposed method, we proved some common fixed point theorems for six mappings by using contractive condition of integral type for class of weakly compatible maps in noncomplete intuitionistic fuzzy metric spaces, without taking any continuous mapping of integral type. The theorems and corollary proved that this research proved the fixed point theorems in terms of mappings in fuzzy metric spaces.

\section{REFERENCES}

Bugajewski, D. and P. Kasprzak, 2009. Fixed point theorems for weakly $\mathrm{F}$-contractive and strongly Fexpansivemappings. J. Math. Anal. Appli., 359: 126-134.
Chauhan, S.S., 2009. Common fixed point theorem for two pairs of weakly compatible mappings in Mfuzzy metric spaces. Int. J. Math. Anal., 3: 393-398.

Cho, Y.J., 1997. Fixed point in fuzzy metric spaces. J. Fuzzy Mathem., 5: 949-962.

Du, W.S., 2012. On coincidence point and fixed point theorems for nonlinear multivalued maps. Topol. Appli., 159: 49-56.

George, A. and P. Veeramani, 1994. On some results in fuzzy metric spaces. Fuzzy Sets Syst., 64: 395-399. DOI: 10.1016/0165-0114(94)90162-7

Grabiec, M., 1988. Fixed point in fuzzy metric spaces. Fuzzy Sets Syst., 27: 385-389.

Jungck, G. and B.E. Rhoades, 2008. Fixed point theorems for occasionally weakly compatible mappings. Erratum, Fixed Point Theory, 9: 383-384.

Klim, D. and D. Wardowski, 2007. Fixed point theorems for set-valued contractions in complete metric spaces. J. Math. Anal. Appli., 334: 132-139. DOI: 10.1016/j.jmaa.2006.12.012

Manro, S., S.S. Bhatia and S. Kumar, 2012. Common fixed point theorems in fuzzy metric spaces. Annals Fuzzy Mathem. Inform., 3: 151-158.

Mishra, M.K., P. Sharma and D.B. Ojha, 2010. Fixed points theorem in fuzzy metric space for weakly compatible maps satisfying integral type inequality. Int. J. Applied Eng. Res., 1: 315-315.

Mishra, R.K. and S. Choudhary, 2010. On fixed point theorems in fuzzy metricspaces. IMACST, 1: 1-1.

Mishra, S.N., N. Sharma and S.L. Singh, 1994. Common fixed points of maps on fuzzy metric spaces. Int. J. Math. Math. Sci., 17: 253-258.

Rhoades, B.E., 1988. Contractive definitions and continuity. Contemporary Math, 72: 233-245.

Schweizer, B. and A. Sklar, 1960. Statistical metric spaces. Pacific J. Math., 10: 313-334.

Singh, D., M. Sharma, R. Sharma and N. Singh, 2012. Some common fixed point theorems in fuzzy metric spaces. Adv. Fixed Point Theory, 2: 92-107.

Zadeh, L.A., 1965. Fuzzy sets. Inform. Control, 89: 338353. 